\title{
The use of urban drainage systems in sustainable cities
}

\author{
M. I. Rodríguez-Rojas ${ }^{1}$, F. Huertas-Fernández ${ }^{1}$, G. Martínez ${ }^{2}$ \\ \& B. Moreno ${ }^{2}$ \\ ${ }^{1}$ Department of Urban and Regional Planning, \\ University of Granada, Spain \\ ${ }^{2}$ Department of Construction and Engineering Projects, \\ University of Granada, Spain
}

\begin{abstract}
Sustainable urban drainage management is of great international importance as a useful tool for the environmental improvement of urban spaces, reducing flooding (increasingly severe and dangerous) and saving on operating costs and expansion of urban sanitation networks. In recent years, the use of 'Sustainable Urban Drainage Systems' or 'SUDS' has become widespread as a new management strategy for sustainable cities. However, there are many uncertainties about what the current SUDS results are, because of the lack of monitoring and control. This is the reason why companies that manage sanitation do not implement more SUDS as suitable solutions. This paper presents a case study developed in the city of Granada, Spain, where some types of SUDS (different permeable pavements) have been built and tested. Information from different episodes of rain and types of SUDS behaviour have been processed. The results show the effectiveness and applicability of these systems, helping to improve sustainability in the city.

Keywords: Sustainable Urban Drainage Systems, SUDS, permeable pavement, sustainable city, floods.
\end{abstract}

\section{Introduction}

Sustainable urban drainage management is becoming increasingly important at an international level, in terms of the environmental improvement of urban spaces, the reduction of flooding (increasingly more serious and dangerous), the reduction of operating costs and the expansion of urban sanitation networks [1]. 
The use of 'Sustainable Urban Drainage Systems' or 'SUDS' [2-4] has become widespread in recent years, being used as a new management strategy which is more in keeping with the principles of sustainability and quality of life [5], trying to restore the natural hydrological cycle which has been altered by the continued impermeability of cities [6]. A multitude of these systems exist (for example, green roofs, rainwater harvesting, permeable pavements, infiltration trenchs and bioretention areas...) [3], but, their main objective is to provide a more sustainable alternative to the management of run-off [7-9]. These systems basically consist of collecting rain water, transporting it and storing it for the maximum time possible, with the aim of slowing it down, and later it is filtered and reused for nonconsumptive use or simply for the refilling of aquifers [3, 4]. The implementation of SUDS has proved to be a very useful tool for Urban Regeneration and Sustainability, advancing towards what has been recently named as 'Water Sensitive Urban Design' (WSUD) [10-12]; the integration of urban planning into the management, protection and conservation of the urban water cycle, in such a way that water management which is sensitive to hydrological and ecological processes is guaranteed, namely, the process of integrating water cycle management with the built environment through planning and urban design [13]. Countries like the United States, the United Kingdom, France and Australia have been using these systems for more than a decade, developing regulations which already require the implementation of SUDS in urban planning [14]. Ultimately, the environmental benefits which are generated by these systems make them a sustainable alternative to traditional urban sanitation management [8].

However, much uncertainty exists concerning the real results of these systems; the lack of monitoring impedes the collection of objective data, necessary for understanding, with exactitude and reliability, their performance and main effects. This makes the introduction of the systems inconvenient, because the companies which manage sanitation do not have enough information to determine their impact and cost efficiency. Therefore, it is necessary for concrete data to be made available in order to help quantify the reduction of flooding risk and the cost of the use of the sanitation networks with regard to urban regeneration and the improvement of sustainability in cities.

Accordingly, this paper presents a case study carried out in the city of Granada, Spain, in which a type of SUDS, permeable pavement, has been experimented with. Information has been collected on its hydrological behaviour in different rain events. The results collected show just how effective and applicable a tool these systems are for the improvement of sustainability in cities.

\section{Objectives}

The main objective of this investigation is to show the results of a case study which has consisted of the integration of different permeable pavements in the city, as a tool for the improvement of urban sustainability. The specific objectives can be summarised as follows:

- Determine the applicability of permeable pavements in cities to:

- $\quad$ Reduce the intensity and frequency of flooding. 
- Improve the performance of the urban sanitation networks and reduce renovation costs

- Increase subterranean hydrological resources, contributing to the improvement of the urban landscape through the creation of new green infrastructure.

- Recognise the efficiency of Permeable Pavements as a Sustainable Urban Drainage System in the city.

- Serve as a reference for the use of permeable pavements in other cities.

\section{Methodology}

This study has been carried out on the Cartuja University Campus, in the city of Granada, in southern Spain (Fig. 1), and has consisted of the construction of a car park in which different permeable pavements have been laid, along with a monitoring system which provides data on the hydrological behaviour of the ground during rain events (Fig. 2). In this paper, the data collected in 2015 has been analysed and processed.
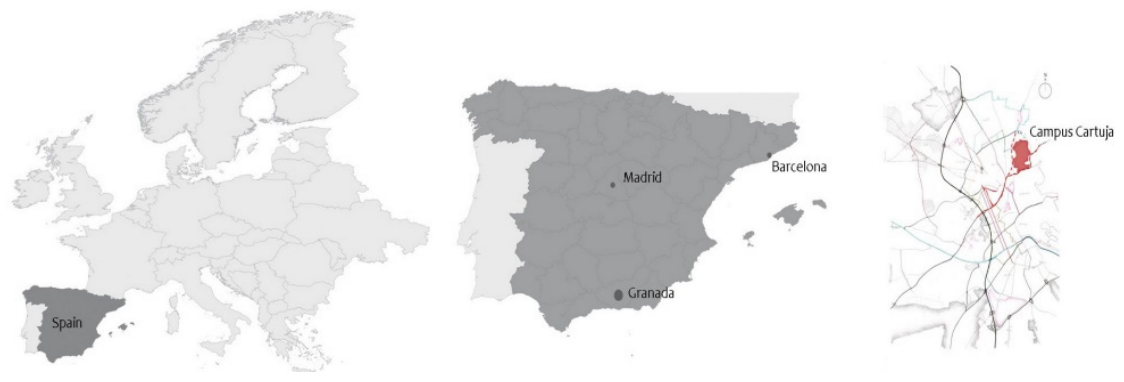

Figure 1: Location of case study.

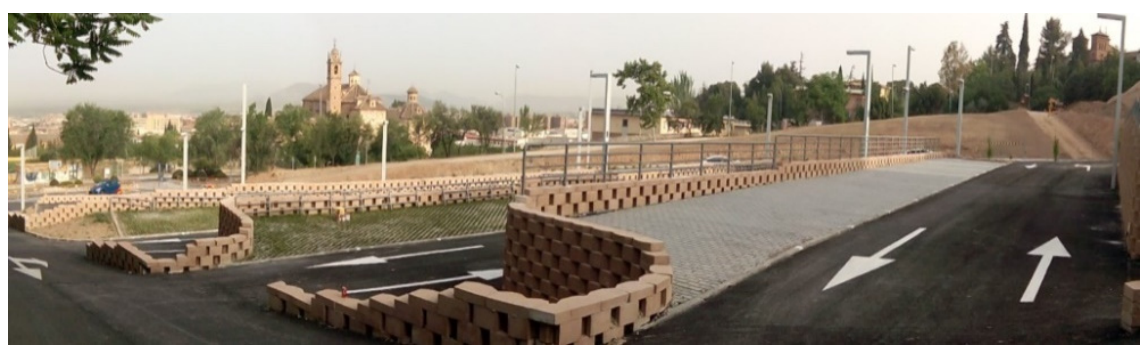

Figure 2: Case study.

The choice of permeable pavements as SUDS to be tested is justified by their high level of applicability in cites, easy integration in a wide typology of spaces, capacity for urban regeneration and high potential for reducing the frequency and intensity of flooding. Three types of permeable pavements have been built used in the parking areas for vehicles (Fig. 3), choosing pavements which can adapt to the 
main urban uses; a Spaced Modular Pavement which can be used in pedestrian areas or areas of slow movement $\left(310 \mathrm{~m}^{2}\right.$ in 3 surface areas), a Grass Grid Pavement which can be used for recreation areas, multi-use areas and/or parking $\left(310 \mathrm{~m}^{2}\right.$ in 2 surface areas) and a Gravel Grid Pavement which can be used in open spaces and/or multifunction areas $\left(196 \mathrm{~m}^{2}\right.$ in 2 surface areas).
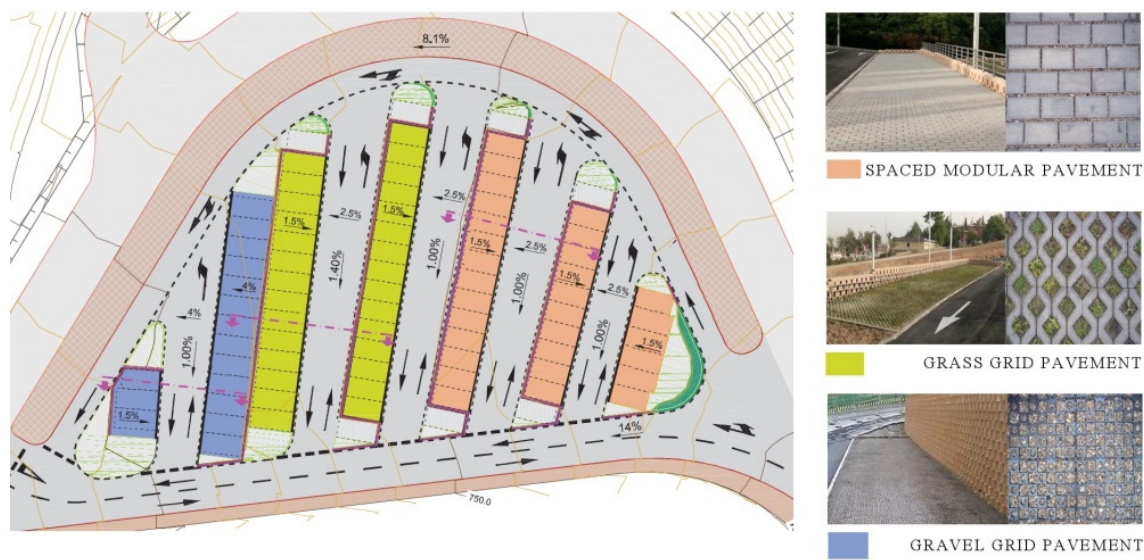

Figure 3: Location of the types of pavement.

The monitoring system consists of a pluviometer which provides data on rainfall volume, a flowmeter installed in each pavement surface which registers water drainage volume, and a data log which stores all the data on a server.

Using all this information and the hydrological model Storm Water Management Model (SWMM) (chosen for its applicability for urban subcatchment), the hydrological behaviour of the tested pavements has been simulated, collecting the following data (Table 1).

Water Residence Time. This variable, related to the retention capacity of the soil, indicates the delay produced by water drainage, which can lead to better performance of sanitation networks.

Peak Flow. The lower this variable is, the lower the maximum volume of water circulating on the street is. This means that the probabilty of flooding is reduced.

Drainage Volume. This variable, which is related to the infiltration capacity of the soil, indicates the amount of water which can get to the aquifer, therefore increasing the available hydrological resources.

In order to assess the environmental improvement, a comparative analysis has been carried out between the hydrological behaviour of the permeable pavements tested and conventional pavements.

For this, Type 0 ground has been defined as having a high level of impermeability (Index $\mathrm{CN}=98$ according to the methodology established by Mishra and Singh [15] and a new simulation has been carried out which obtains the previously mentioned data for the conventional pavement. 
Table 1: Data analysis.

\begin{tabular}{|c|c|c|}
\hline \multicolumn{3}{|c|}{ DATA PROVIDED BY THE MONITORING SYSTEM } \\
\hline \multicolumn{2}{|c|}{ DATA } & DEFINITION \\
\hline \multicolumn{2}{|c|}{ Rainfall volume } & Rainfall volume in a rain event \\
\hline \multicolumn{2}{|c|}{ Drainage volume } & Storm water runoff drains \\
\hline \multicolumn{3}{|c|}{ DATA OBTAINED WITH SWMM } \\
\hline $\begin{array}{l}\text { PERMEABLE } \\
\text { PAVEMENTS } \\
(\text { TYPE } 1,2,3)\end{array}$ & $\begin{array}{c}\text { CONVENTIONAL } \\
\text { PAVEMENT } \\
\text { (TYPE 0) }\end{array}$ & DEFINITION \\
\hline $\begin{array}{l}\text { Water residence time } \\
\qquad\left(T_{\text {SuDS }}\right)\end{array}$ & $\begin{array}{l}\text { Water residence time } \\
\qquad(\text { TTYPE } 0)\end{array}$ & $\begin{array}{l}\text { Time taken by the soil to drain all } \\
\text { the rain water during a rain event }\end{array}$ \\
\hline $\begin{array}{l}\text { Peak flow } \\
\text { (FsuDs) }\end{array}$ & $\begin{array}{l}\text { Peak flow } \\
(\text { FTYPE } 0)\end{array}$ & $\begin{array}{l}\text { Maximum flow generated by a } \\
\text { rain event in a watershed }\end{array}$ \\
\hline $\begin{array}{l}\text { Drainage volume } \\
\text { (VSUDS) }\end{array}$ & $\begin{array}{l}\text { Drainage volume } \\
\quad\left(\mathrm{V}_{\text {TYPE } 0)}\right.\end{array}$ & $\begin{array}{c}\text { Soil water Infiltration in a rain } \\
\text { event }\end{array}$ \\
\hline
\end{tabular}

A series of efficiency parameters has been defined for all of the pavements (Types 0, 1, 2 and 3), from the hydrological information which was obtained. These parameters quantify the improvement produced by each type of permeable pavement when compared to the conventional type. The parameters also define the percentage of variation compared with the variables obtained through SWMM (Table 2). The analysis of these efficiencies shows the main results of the behaviour of the tested pavements.

Table 2: Defined efficiency parameters.

\begin{tabular}{|c|c|c|}
\hline \multicolumn{3}{|c|}{ EFFICIENCY PARAMETERS (\%) } \\
\hline $\begin{array}{c}\text { Water residence } \\
\text { time efficiency } \\
\left(\boldsymbol{\varepsilon}_{\mathbf{T}}\right)\end{array}$ & $\begin{array}{c}\text { Increase in Water Residence } \\
\text { Time generated by the } \\
\text { permeable pavement compared } \\
\text { with a conventional pavement }\end{array}$ & $\varepsilon \mathbf{t}=\left(\frac{\boldsymbol{T}_{\text {SUDS }}-\boldsymbol{T}_{\text {Type } \mathbf{0}}}{\boldsymbol{T}_{\text {SUDS }}}\right) * \mathbf{1 0 0}$ \\
\hline $\begin{array}{c}\text { Peak flow } \\
\text { efficiency } \\
\left(\boldsymbol{\varepsilon}_{\mathbf{F})}\right)\end{array}$ & $\begin{array}{c}\text { Reduction in Peak Flow } \\
\text { generated by the permeable } \\
\text { pavement compared with a } \\
\text { conventional pavement }\end{array}$ & $\varepsilon \mathbf{f}=\left(\frac{\boldsymbol{F}_{\text {Type 0 }}-\boldsymbol{F}_{\text {SUDS }}}{\boldsymbol{F}_{\text {Type 0 }}}\right) * \mathbf{1 0 0}$ \\
\hline $\begin{array}{c}\text { Drainage volume } \\
\text { efficiency } \\
\left(\boldsymbol{\varepsilon}_{\mathbf{v}}\right)\end{array}$ & $\begin{array}{c}\text { Increase in Drainage Volume } \\
\text { generated by the permeable } \\
\text { pavement compared with a } \\
\text { conventional pavement }\end{array}$ & $\varepsilon \mathbf{v}=\left(\mathbf{1}-\frac{\boldsymbol{V}_{\text {SUDS }}}{\boldsymbol{V}_{\text {Type } \mathbf{0}}}\right) * \mathbf{1 0 0}$ \\
\hline
\end{tabular}




\section{Results}

The results which have been obtained using the previous methodology can be summarised as follows:

\section{Analysis of the types of efficiency of permeable pavements in comparison} with conventional pavements. For example, Table 3 shows the results which were obtained for 3 representative rain events (low, medium and high Rainfall Volume values with regard to all the registered events), and in figure 4, all of the average data obtained for each type of pavement in all of the registered rain events. In both tables, very favourable results can be seen for the three variables which have been analysed.

Water residence time efficiencies. As can be seen in Table 3, these efficiencies are higher during low and medium level rain events, which indicates that efficiency worsens with increased Rainfall Volume, therefore characterising the most suitable climatology for the installation of these types of pavement. However, as can be seen in figure 4, average values close to $50 \%$ have been obtained, demonstrating that these types of pavements can retain water for a long time, considerably improving the performance of the sanitation networks and reducing maintenance and renovation costs.

Peak flow efficiencies. The values obtained which are over $95 \%$ indicate that the maximum flow is $5 \%$ of what is generated by a conventional solution. This fact represents great potential for reducing the risk of flooding in urban areas.

Drainage volume efficiencies. These efficiencies show great variation concerning pavement and types of rain. This may well be due to the influence of the initial humidity conditions of the soil, which indicates the need to carry out a new study to consider the initial saturation of the ground as a variable to be analysed. However, the average values of around $45 \%$ indicate a considerable increase in infiltration, which will generate a sizable contribution to the aquifer, which is essential for the increase in subterranean hydrological resources and the creation and maintenance of new green infrastructure. Said average value goes down to $35 \%$ for type 2 , with the lowest value for medium and high rain events (as 23\% and 10\% respectively). This makes it the type of pavement which shows the lowest capacity for infiltration and the highest level of water retention. 
Table 3: Efficiencies obtained for 3 rain events in the three types of permeable pavements.

\begin{tabular}{|c|c|c|c|c|}
\hline Rainfall volume (mm) & Type of pavement & $\boldsymbol{\varepsilon}_{\mathbf{T}} \mathbf{( \% )}$ & $\boldsymbol{\varepsilon}_{\mathbf{F}} \mathbf{( \% )}$ & $\boldsymbol{\varepsilon}_{\mathbf{V}} \mathbf{( \% )}$ \\
\hline \multirow{3}{*}{7} & 1 & 65 & 95 & 55 \\
\cline { 2 - 5 } & 2 & 73 & 98 & 74 \\
\cline { 2 - 5 } & 3 & 60 & 97 & 32 \\
\hline \multirow{3}{*}{18} & 1 & 57 & 95 & 68 \\
\cline { 2 - 5 } & 2 & 48 & 99 & 23 \\
\cline { 2 - 5 } & 3 & 46 & 95 & 70 \\
\hline \multirow{3}{*}{32} & 1 & 29 & 98 & 32 \\
\cline { 2 - 5 } & 2 & 36 & 99 & 10 \\
\cline { 2 - 5 } & 3 & 29 & 96 & 43 \\
\hline
\end{tabular}

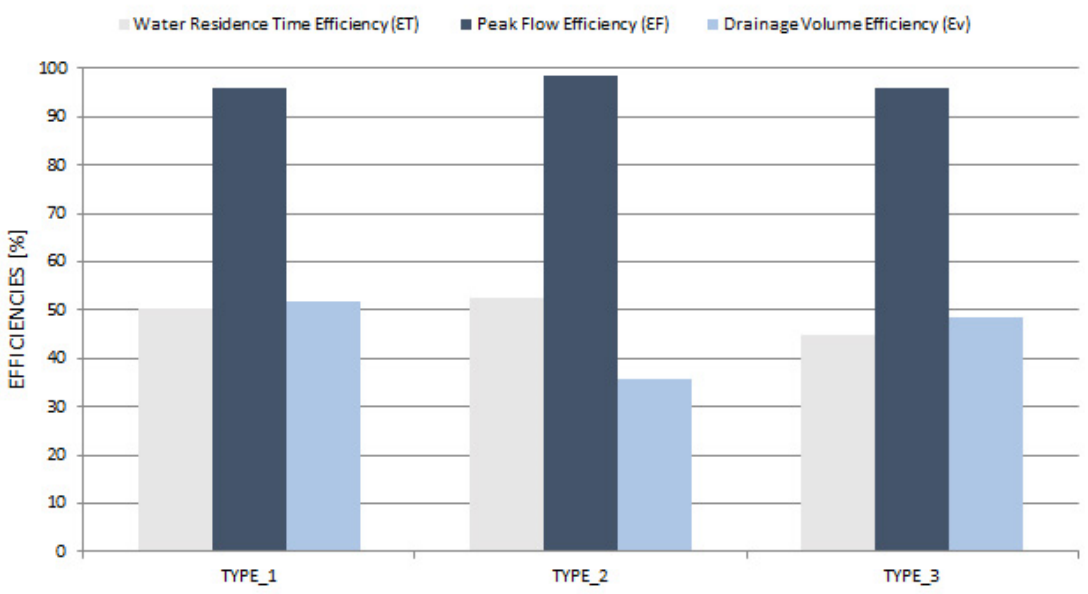

Figure 4: Average efficiencies for the registered rain events.

2. The second result which has been obtained is the comparative hydrograph of the permeable pavements in comparison with conventional pavements. For example, figures 5, 6 and 7 show the enormous Flow decrease produced in the 3 permeable pavements during the rain event of Rainfall Volume $=18 \mathrm{~mm}$, as well as an important delay in drainage time, which can be understood, as before, in very favourable efficiency values.

Both results show an extremely significant improvement in the hydrological behaviour of the ground, presenting permeable pavements as a more sustainable alternative solution to conventional sanitation systems. 
782 Sustainable Development and Planning VIII

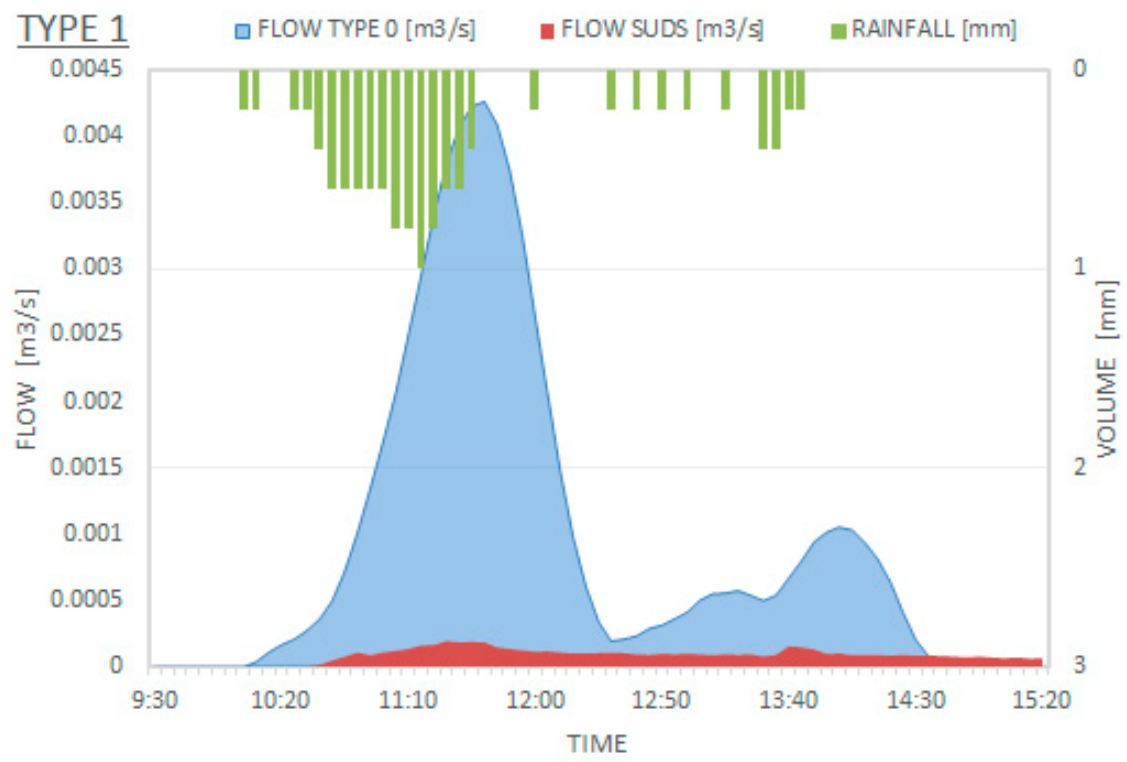

Figure 5: Comparative Hydrograph Conventional Solution/SUDS Type 1.

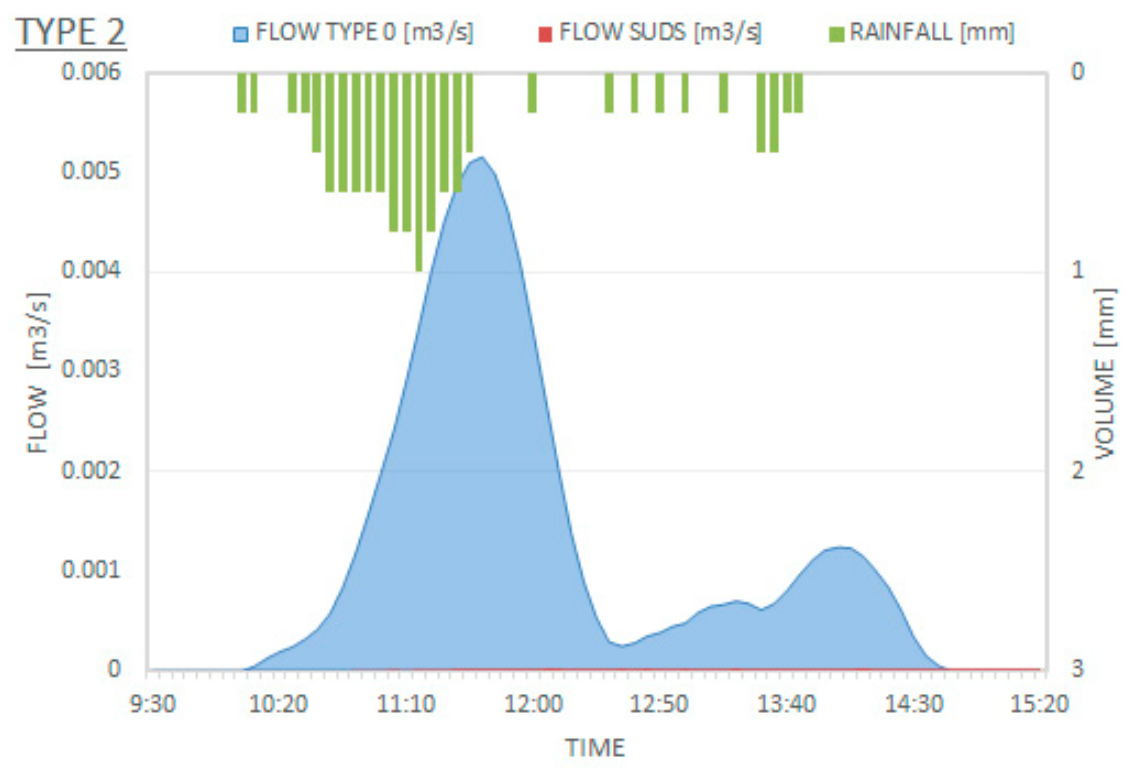

Figure 6: Comparative Hydrograph Conventional Solution/SUDS Type 2. 


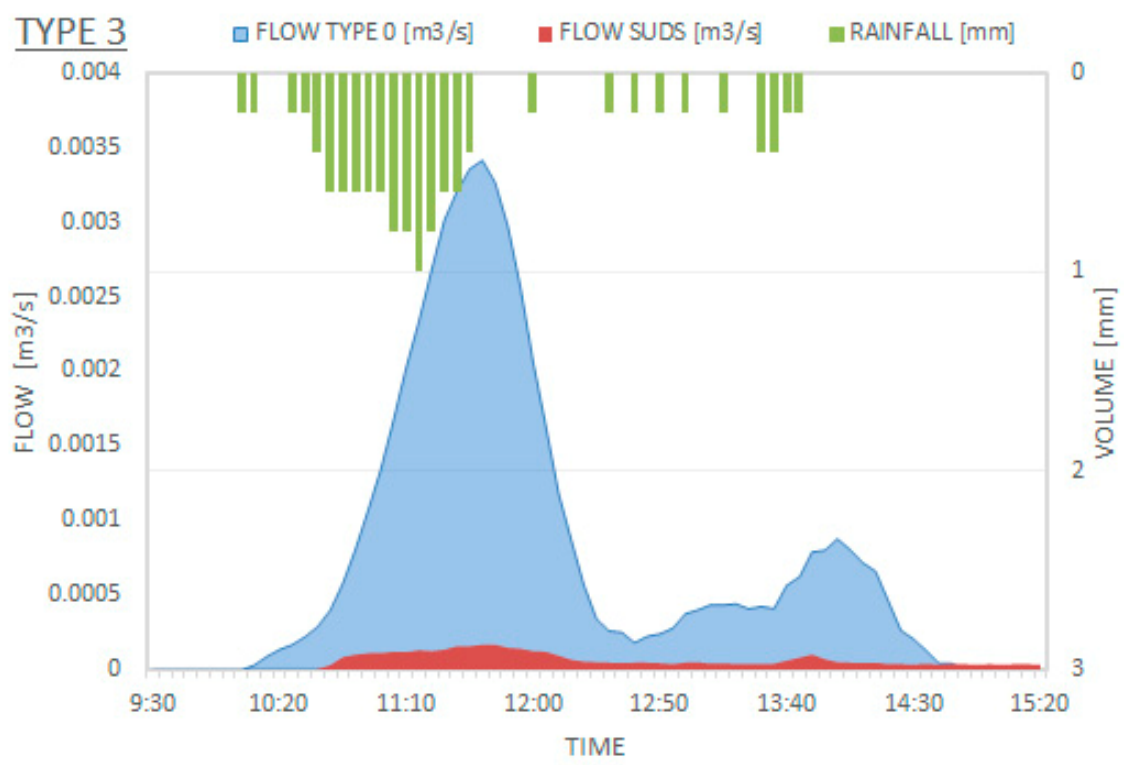

Figure 7: Comparative Hydrograph Conventional Solution/SUDS Type 3.

\section{Conclusions}

From the results obtained by this research, the conclusion can be drawn that type 2 pavements produce better average efficiency values when compared to type 1 and 3 pavements, which generate somewhat lower data, which is quite similar in each of the two cases. However the behaviour of the pavements is better overall for low and medium level rain events, indicating that their performance worsens with an increase in Rainfall Volume. Finally the average efficiency values which have been reached (50\% for Water Residence Time, 95\% for Peak Flow and $45 \%$ for Drainage Volume), show the great efficiency of these systems and their functionality as an alternative to conventional sanitation networks.

These results fulfill the objectives set out in this research, enormously reducing the intensity and frequency of flooding, considerably improving the performance of urban sanitation networks and greatly increasing subterranean hydrological resources. Due to this, it is evident that the integration of these types of pavements is suitable as a tool for the improvement of urban sustainability and the integration of water management and urban planning. Accordingly, this case study is of an innovative character and is very relevant for the field of water engineering and planning as it is an important advance in the integration of SUDS in cities and in Water Sensitive Urban Design. 


\section{Acknowledgements}

The authors would like to thank the European Union for financial support via the project "Gestión Sostenible de Aguas Pluviales en Zonas Urbanas" of the "Programa Operativo FEDER de Andalucía 2007-2013". We would also like to thank the AOPJA staff and researchers for their dedication and professionalism.

\section{References}

[1] Rodríguez, M., Cuevas, M., Martínez, G., \& Moreno, B. Planning criteria for Water Sensitive Urban Design. WIT Transactions on Ecology and the Environment (191), 1579-1591, 2014.

[2] Charlesworth, S., Harker, E., \& Rickard, S. A Review of Sustainable Drainage Systems (SuDS): A Soft Option for Hard Drainage Questions? Geography, 88, 99, 2003.

[3] CIRIA, C. The SUDS Manual. London: CIRIA., 2007.

[4] Zhou, Q. A Review of Sustainable Urban Drainage Systems Considering the Climate Change and Urbanization Impacts. Water, 6, 976-992, 2014.

[5] Prokop, J., Jobstmann, H., \& Schönbauer, A. Overview on best practices for limiting soil sealing and mitigating its effects in EU-27, 2011.

[6] Technische Commissie Bodem. Advisory report on general conditions for soil sealing in urban areas. TCB A063, The Hague and references therein, 2010.

[7] Hoùsková, B., \& Montanarella, L. The natural susceptibility of European soils to compaction. Threats to Soil Quality in Europe. European Commission, Joint Research Centre, 23-36, 2008.

[8] Rodríguez, M., Cuevas, M., F., H., G., M., \& B., M. Indicators to evaluate Water Sensitive Urban Design in Urban Planning. WIT Transactions on Ecology and the Environment (168(1)), 371-382, 2015.

[9] SWD. Guidelines on best practice to limit, mitigate or compensate soil sealing. Comminssion Staff Working Document, 2012.

[10] Wong, T. An overview of Water Sensitive Urban Design practices in Australia. Water Prac. Technol. 1 (1). 2006.

[11] Brown, R., \& Clarke, J. Transition to Water Sensitive Urban Design: The Story of Melbourne, Australia. Melbourne: Facility for Advancing Water Biofiltration, Monash University, 2007.

[12] Joint Steering Committee for Water Sensitive Citie. Evaluating Options for Water Sensitive Urban Design-A National Guide. Canberra: Australian Government (National Water Commission), 2009.

[13] CIRIA, C. Planning for SUDS-making it happen. London: CIRIA, 2010.

[14] CIRIA, C. Water Sensitive Urban Design in UK. London: CIRIA, 2013.

[15] Mishra, S.K.; Singh, V., Soil Conservation Service Curve Number (SCSCN) Methodology, Water Science and Technology Library, 42, 2003. 